Communications in Physics, Vol. 20, No. 3 (2010), pp. 265-270

\title{
MATCHING OF PUMPED AND LASER MODE VOLUMES INSIDE A DIODE SIDE-PUMPED SOLID-STATE LASER
}

\author{
HO QUANG QUY \\ Academy of Military Science and Technology \\ MAI VAN LUU AND VU NGOC SAU \\ Vinh University
}

\begin{abstract}
The condition to match the pumped-volume's width with the laser mode-volume's waist in a diode four side pumped solid-state laser is analyzed by numeric solutions of the approximation equation. As a result, the dependences of pumped volume's width on pumping diode parameter and laser mode volume's waist on cavity parameter were found. Such a matching condition was simultaneously determined by cavity, pumping beam and laser rod parameters.
\end{abstract}

\section{INTRODUCTION}

The diode side-pumped solid-state laser is one of advanced lasers and being paid much attention in $[1,2]$. High laser efficiency can be obtained because there is a good spetial match between the emission spectrum of pump beam and the absorption spectrum of solid-state laser medium. The optimum relation between the the pumped and the mode volumes was obtained by theoretical analysis and experiment, which includes thermally induced diffraction loss [3]. A good spatial match between the pumped and the laser mode volumes can be obtained by using the end-pumping scheme, but the pump energy decreases exponentially along the axial direction. Side-pumping geometry was used to achieve high output laser powers [2]. For laser oscillator, one might use a near-Gaussian pumping intensity distribution centered around the rod axis to help achieve $T E M_{00}$ output; for amplifier, one might use illumination to achieve the best radial uniformity [5]. In the work [4], the relationship between the size of pump beam and mode volume (MV) was analyzed by using space-dependent rate equations, which include the thermally induced diffraction loss. In the work [6], we have shown that the excited active centers (EAC) in the ovelapping area of pump intensities in a four sides configuration are spatially distributed as near-Gaussian function. Moreover, in the work [7], we concluded that a suitable parameter collection of laser diode's beams (sparseness, location and azimuthal angle) could be chosen so that the longitudinal distribution of EAC in the laser rod is homogeneous. Thus, by four-side pumping, the laser rod is seem to be a graded-EAC rod with its EAC volume of certain width. If EACV width matches with MV waist the pump efficiency is enhanced. But, the matching condition depends on many parameters of the pump laser diodes and the cavity.

In this work, we introduce the approximation equation describing the matching condition between EACV width and MV waist. In. Sec. II, we investigate the dependence 
of EACV width on pumping laser beam and that of MV waist on cavity parameters. Finaly, the matching condition and its suitable parameter collection are discissed.

\section{APPROXIMATION EQUATION FOR SPATIAL MATCHING BETWEEN EACV WIDTH AND MV WAIST}

As shown in previous work [6], the spatial distribution of four-side-pump intensity in overlapping area of laser rod's cross-section is a near-Gausssian function. It means that EAC's density distribution in cross-section of laser rod is a near-Gaussian function of radial distance too. With this assuming, the distribution of average EAC's density can be given by the following function

$$
Q_{\text {fourside }}(x, y)=B N \frac{I_{\text {fourside }}(x, y)}{h \bar{\nu}}=Q_{E A C 0} \exp \left[-\frac{2\left(x^{2}+y^{2}\right)}{W_{E A C}^{2}}\right],
$$

where $B$ is the Einstein's coefficient, $N$ is the density of active centers in laser rod, $Q_{E A C 0}$ is the maximum EAC's density in rod axis, $W_{E A C}$ is the width of EACV, $h \bar{\nu}$, and $I_{\text {fourside }}(x, y)$ intensity of exciting field, which is given in work [6] as follows:

$$
\begin{aligned}
I_{\text {fourside }}(\rho)= & I_{\text {fourside }}(x, y) \\
= & I_{0} W_{i n 0}^{2}\left\{\left[\frac{1}{W_{i n}(y)}\right]^{2} \exp \left[\frac{-2 x^{2}}{W_{i n}^{2}(y)}\right]+\left[\frac{1}{W_{i n}(-y)}\right]^{2} \exp \left[\frac{-2 x^{2}}{W_{i n}^{2}(-y)}\right]\right. \\
& \left.+\left[\frac{1}{W_{i n}(x)}\right]^{2} \exp \left[\frac{-2 y^{2}}{W_{i n}^{2}(x)}\right]+\left[\frac{1}{W_{i n}(-x)}\right]^{2} \exp \left[\frac{-2 y^{2}}{W_{i n}^{2}(-x)}\right]\right\},
\end{aligned}
$$

where all parameters of pump beam inside laser rod are given as:

$$
\begin{aligned}
& W_{i n o}=M W_{0} \text { is the beam waist inside laser rod, } \\
& b_{i n}=M^{2} b \text { is the confocal parameter inside laser rod, } \\
& W_{i n}(x)=W_{i n 0}\left[1+\left(\frac{x}{b_{i n}}\right)^{2}\right]^{\frac{1}{2}} \text { is the width at } \mathrm{x} \text { inside laser rod, } \\
& M=\frac{M_{t}}{\sqrt{1+t}} ; t=\frac{r_{0}}{y_{0}-r_{0} /(n-1)} ; M_{t}=\left|\frac{\mid}{y_{0}(n-1)-r_{0}}\right|
\end{aligned}
$$

$r_{0}$ is the laser rod radiur, $y_{0}$ is the distance between laser rod axis and $w_{0}$ pump beam's waist, $b$ is the confocal parameter of pumping beam, $n$ is the refractive index of laser rod. All above parameters are illustrated in Fig. 1.

Assuming four pumping laser diode arrays are symmetrical through rod axis, (2) can be simplified to

$$
I_{\text {fourside }}(\rho)=4 I_{0}\left\{\left[\frac{W_{i n 0}}{W_{i n}}(\rho)\right]^{2} \exp \left[-\frac{2 \rho^{2}}{W_{i n}^{2}}(\rho)\right]\right\}=4 I_{0} G(\rho),
$$

where $\rho=\sqrt{x^{2}+y^{2}}$ is the radial radius from rod axis. 


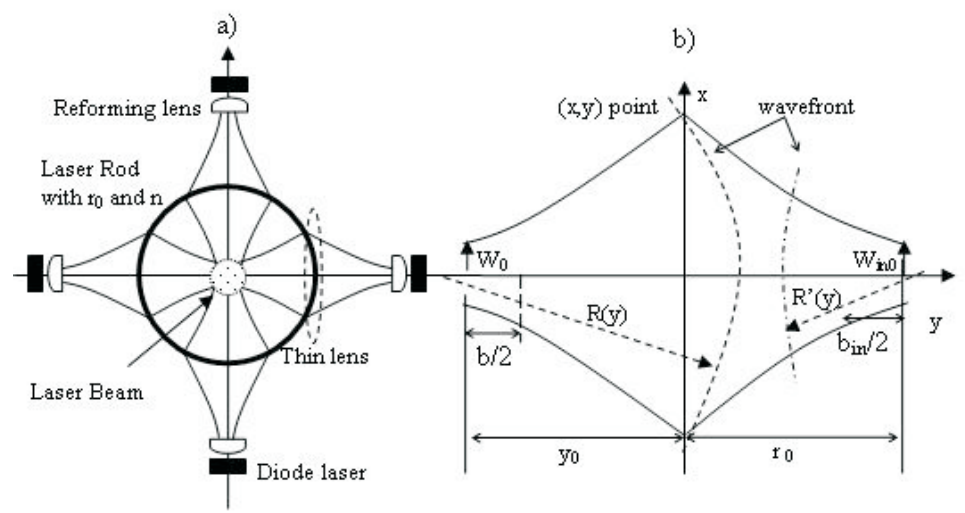

Fig. 1. a- Cavity geometry for four side-pumping module; b- Cross-sectional geometry of Gaussian beam outside and inside laser rod.

From $(1),(3),(4),(5),(6)$ and $(7)$, the width of EACV is given by:

$$
W_{E A C}=\sqrt{-\frac{2 \rho^{2}}{\ln \left(\frac{B N I_{\text {fourside }}(\rho)}{h \bar{\nu} Q_{E A C 0}}\right)}}=\sqrt{-\frac{2 \rho^{2}}{\ln \left(\frac{B N I_{0}}{h \bar{\nu} Q_{E A C 0}}\right)}+\ln (G(\rho))} .
$$

Since the maximum EAC density, $Q_{E A C 0}$ depends on the maximum intensity $I_{0}$, so it can be chosen so that the first term in denominator under square-root to be zero and (8) is reduced to

$$
W_{E A C}=\sqrt{-\frac{2 \rho^{2}}{\ln (G(\rho))}} .
$$

As shown in work [6], the distribution of overlopping intensity is near-Gaussian with certain width (Fig. 2).

We approximately consider that pump intensity higher than contributes to exciting process. Thus, the width of pump intensity distribution can be chosen as root of the following equation

$$
\left\{\left[\frac{W_{i n 0}}{W_{i n}(\rho)}\right]^{2} \exp \left[-\frac{2 \rho^{2}}{W_{i n}^{2}(\rho)}\right]\right\}-1 / e=0
$$

and is noted as (root of equation (10)-RE)

$$
R E\left(\rho_{1} / e\right) \text {. }
$$

Substituting (10)and (11) into (9), we have

$$
W_{E A C}=R E\left(\rho_{1} / e\right) .
$$

From $(3),(4),(5),(6),(10),(11)$ and (12), we can see that the width of EACV depends on $r_{0}, y_{0}, n$ and the wavelength of pumping laser $\lambda_{p}$ (in term of confocal parameter $b$ ). Now, 


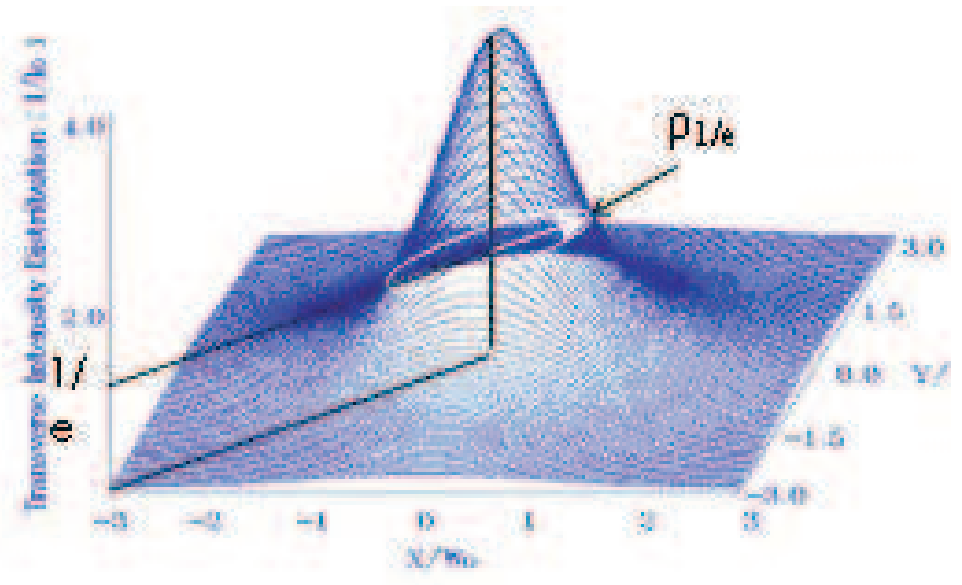

Fig. 2. Four-side-pump intensity distribution inside the laser rod [6]. $\rho_{1 / e}$ is the width at $I_{\max }=4 I_{0} / e$.

we consider the EACV is placed in a confocal cavity of length, L and mirror's curvature radius, $\mathrm{R}$. This cavity has mode $\mathrm{TEM}_{00}$, whose $\mathrm{MV}$ waist, $W_{M O D E}$ is given by [8]

$$
W_{M O D E}=\sqrt{\frac{\lambda_{L}}{2 \pi} \sqrt{L(R-L)}}
$$

where $\lambda_{L}$ is the laser wavelength. From (10), (11), (12) and (13), the matching condition is described by following equation

$$
\left.W_{E A C}=W_{M O D E} \text { or } R E\left(\rho_{1} / e\right) \equiv W_{E A C}\right)=\sqrt{\frac{\lambda_{L}}{2 \pi} \sqrt{L(R-L)}} .
$$

The matching-condition equation (14) contains a single "called" unknown variable $W_{E A C}$. Note that the left hand side (LHS), which is characterized by a "called" pumping parameter $-W_{E A C}$, depends on the parameters of laser diode arrays and laser rod, and the right hand side (RHS), which is characterized by a "called" cavity parameter- $W_{M O D E}$, depends on that of the cavity. If $W_{E A C}=W_{M O D E} \equiv W$, i.e. matching condition satisfies, the equation (14) may be graphically solved by plotting its LHS and RHS versus $W$ and finding the intersections. Every intersection point corresponds to $W$ with a distinct collection of pumping and cavity parameters.

\section{SIMULATED RESULTS AND DISCUSSION}

We consider a solid-state laser generating at the wavelength of $1.06 \mu \mathrm{m}$ pumped by four $0.83 \mu \mathrm{m}$ laser diodes. The spatial distribution of pumping laser diodes is assumed a Gaussian beam characterized by a waist $W_{0}=\left(5 \times 10^{-2}\right.$ and $\left.10.10^{-2}\right) \mathrm{mm}$. The laser rod has its refractive index, $\mathrm{n}=1.78$, and its radius of $4.0,3.0,2.0,1.5$ and $1.0 \mathrm{~mm}$. The location of the pump beam is changed from $1 \mathrm{~mm}$ to $20 \mathrm{~mm}$, and the curvature radius of the mirror is changed from $1 \mathrm{~m}$ to $20 \mathrm{~m}$. 

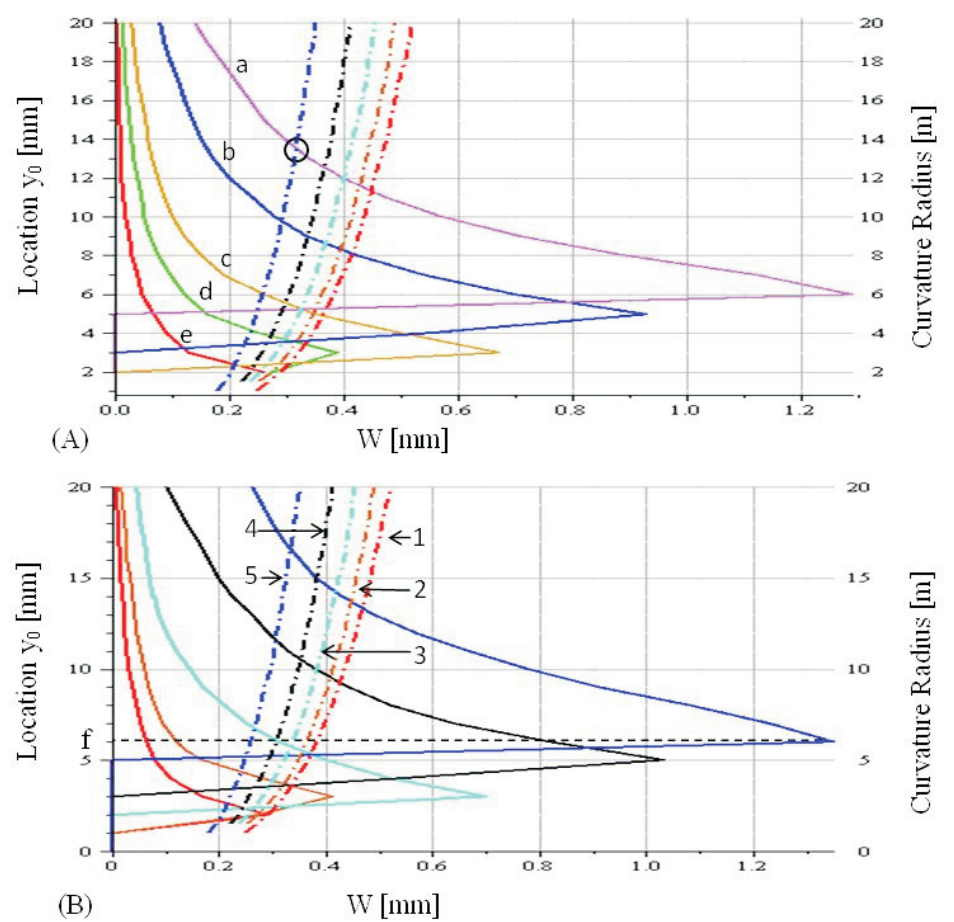

(B)

$\mathrm{W}[\mathrm{mm}]$

Fig. 3. Graphical construction for solving the matching-condition equation (10). The left- and right-hand sides are plotted as function. The intersection points are the solutions. (A) $W_{0}=5.10^{-2} \mathrm{~mm}$ and (B) $W_{0}=10.10^{-2} \mathrm{~mm}$.

As illustrated in Fig. 3 (A) for $W_{0}=5.10^{-2} \mathrm{~mm}$ and Fig. 3 (B) for $W_{0}=10.10^{-2} \mathrm{~mm}$, the LHS has five branches corresponding to the laser rod radiurs, $r_{0}=4.0(\mathrm{a}), 3.0(\mathrm{~b})$, 2.0(c), 1.5(d) and 1.0(e) mm and the RHS has five branches corresponding to the cavity lengths, $L=20(5), 40(4), 60(3), 80(2)$, and 100(1) mm, respectively. From Fig. 3, we can see that: first, for every LHS branch there are many RHS branches that the matching condition satisfies; second, to have larger width $W_{E A C}$, the pumping beam waist of laser diode should be located near the foci of laser rod, i.e., $y_{0} \approx f=r_{0} /(n-1)$ corresponds to that the cavity length and the curvature mirror radius must be chosen longer as possible; third, every intersection point corresponds to a matching volume with distinct collection of parameters, for example, to have an intersection point marked by circle, a collection of $y_{0}=13.5 \mathrm{~mm}, r_{0}=4.0 \mathrm{~mm}, W_{0}=5.10^{-2}, L=20 \mathrm{~mm}$, and $R=13.75 \mathrm{~m} \ldots$ must be chosen. Finally, the matching of pump volume (or EAC volume) with mode volume inside laser rod will be appeared when a collections of pumping parameters $\left(W_{0}, y_{0}, \lambda_{p}\right)$ and cavity parameters $\left(L, R, \lambda_{L}\right)$ are chosen suitably with a given collection of laser rod parameters $\left(r_{0}, n\right)$. 


\section{CONCLUSION}

The approximation equation describing the spatial matching condition between the EACV width and the MV waist of a solid-state laser pumped by four-side laser diodes is considered. This equation is numerically resolved to find the pumping parameter-EACV's width and cavity-MV's waist characteristics. The intersection points of those characteristical curves are the matching values. Based on the matching values, the distinct collection of main parameters of pumping laser diodes (beam waist, location) and cavity (length, curvature radius) can be suitably chosen to enhance pumping efficiency.

\section{REFERENCES}

[1] B. J. Comaskey, et al., IEEE J. Quant. Electron. 28 (1992) 992-996.

[2] N. Hodgson, S Dong, and Q. Lu, Opt. Lett. 18 (1993) 1727-1729.

[3] R. J. St. Pierre et al, IEEE J. Sel.Top. Quant. Electron. 3 (1997) 53-58.

[4] W. Xie et al., Applied Optics, 39 (2000) 5482-5487.

[5] T. Y. Fan, and R. L. Byer, IEEE J. Quant. Electron. 24 (1988) 895-912.

[6] H. Q. Quy et al., Commun. in Phys. 18 (2009) 39-44.

[7] H. Q. Quy et al., Advances in OPS and Appl. (2009) 237-241.

[8] A. M. Prochorov, Laser Handbook, V.2, Sovietskoe Radio, Moscow, 1978.

Received 30 September 2009. 